\title{
Philosophiques
}

\section{Sophie Bourgeault et Julie Perreault (dir.) Le care. Éthique féministe actuelle, Montréal, Les Éditions du Remue-ménage, 2015, 280 pages}

\section{Cécile Gagnon}

Volume 44, numéro 2, automne 2017

URI : https://id.erudit.org/iderudit/1042349ar

DOI : https://doi.org/10.7202/1042349ar

Aller au sommaire du numéro

Éditeur(s)

Société de philosophie du Québec

ISSN

0316-2923 (imprimé)

1492-1391 (numérique)

Découvrir la revue

Citer ce compte rendu

Gagnon, C. (2017). Compte rendu de [Sophie Bourgeault et Julie Perreault (dir.) Le care. Éthique féministe actuelle, Montréal, Les Éditions du Remue-ménage, 2015, 280 pages]. Philosophiques, 44(2), 420-424.

https://doi.org/10.7202/1042349ar

Ce document est protégé par la loi sur le droit d'auteur. L'utilisation des services d'Érudit (y compris la reproduction) est assujettie à sa politique d'utilisation que vous pouvez consulter en ligne.

https://apropos.erudit.org/fr/usagers/politique-dutilisation/ 


\section{Conclusion: Entre écrits populaires et écrits techniques}

La lecture de Solger est loin d'être aisée. Bien que précis et rigoureux, le vocabulaire solgérien reste technique et demande qu'on lui prête attention. La présente édition critique conviendra surtout aux spécialistes, notamment en raison de ses multiples passages polémiques. Néanmoins, étant donné son exigence dialogique et ses multiples renvois surplombant les problématiques centrales de son temps, les écrits de Solger peuvent se révéler être une excellente introduction à tous ceux qui s'intéressent de près à la philosophie allemande classique, pour peu que l'intéressé(e) sache faire preuve d'une patience digne du concept.

ANDREAS FARINA-SCHROLL

Université de Montréal

\section{Sophie Bourgeault et Julie Perreault (dir.) Le care. Éthique féministe actuelle, Montréal, Les Éditions du Remue-ménage, 2015, 280 pages.}

L'ouvrage collectif dirigé par Sophie Bourgeault et Julie Perreault a pour objectif de poser les bases conceptuelles de ce que les auteures qualifient de "deuxième vague» de l'éthique du care. Elles nous proposent une lecture dé-genrée et dé-sentimentalisée du care - s'inscrivant dans le prolongement des écrits de Joan Tronto - afin de positionner clairement l'éthique du care comme théorie critique féministe. Débarrassé de ses tendances essentialistes, le care devient dès lors un outil pour renouveler les concepts actuels de démocratie et de justice.

L'intérêt particulier de l'ouvrage est l'application du potentiel critique de l'éthique du care aux enjeux politiques et sociaux propres au contexte québécois ainsi qu'à des questions philosophiques contemporaines. Pour ce faire, on nous présente la théorie réactualisée du care de façon à pouvoir ensuite en développer la force réflexive sur deux axes d'analyse: politique et philosophique. Cette structure permet de mieux saisir l'intérêt de cette théorie éthique relativement jeune, mais également d'en comprendre et d'en apprécier la portée tant pratique que théorique. Il s'agit donc d'un ouvrage à la fois d'introduction et d'approfondissement dont la division en trois temps permet une progression logique accessible.

Dans la partie «Relecture féministe", les auteures nous présentent une notion élargie du care basée sur une relecture dé-genrée qui ne peut plus se butter aux critiques d'essentialisme ou de simple sentimentalisme. Julie Perreault propose, pour ce faire, une relecture des premiers écrits de Carol Gilligan de façon à en révéler le potentiel théorique, sans toutefois en nier les limites. Elle souhaite ainsi rallier pertinemment les concepts de justice et de care afin de faire de l'éthique du care le point d'ancrage d'une phénoménologie féministe. 
Ensuite, Patricia Paperman aborde la question de la division actuelle du travail universitaire entre les approches descriptives et les approches normatives, division qui fragmente, selon elle, les connaissances sur l'éthique du care et, conséquemment, invisibilise la théorie. Lorsque nous posons la question "Qui s'occupe de quoi et comment?", Paperman nous invite à nous attarder à l'expérience particulière sans dissocier la description de l'évaluation que nous en faisons. Cette réflexion nous permet de réaliser que la seule manière de développer à son plein potentiel l'éthique du care est de sortir du cadre d'analyse philosophique de l'éthique afin d'intégrer à nos réflexions les cadres conceptuels et analytiques des sciences sociales.

Le chapitre de Naïma Hamrouni clôt cette première partie en proposant une redéfinition du care et de la dépendance afin de mettre fin à l'invisibilité politique des soins. Hamrouni remarque que notre incapacité actuelle à questionner les injustices découlant de la division genrée du travail du care nous amène à adopter des politiques publiques de soins qui cristallisent l'attribution des tâches de soutien aux femmes (et aux autres groupes marginalisés) en plus de réaffirmer la dévaluation des soins. Hamrouni nous propose donc de redéfinir les responsabilités de chaque citoyen ainsi que les conditions de participation à la vie démocratique à travers une reconnaissance politique de l'importance des relations de soins et des individus qui y prennent part. Ce chapitre est particulièrement intéressant puisque Hamrouni y expose clairement le potentiel critique féministe de l'éthique du care ainsi que sa nécessaire politisation.

Dans la deuxième partie de l'ouvrage, dont le thème est «Le care comme outil critique pour repenser le libéralisme", il est question de la portée politique de la compréhension renouvelée du care. En nous présentant l'application pratique de l'éthique du care à des enjeux sociaux actuels, les auteures explicitent et situent le potentiel réformateur de la théorie. À partir de la réflexion théorique de la première partie, on tente de répondre à certains enjeux politiques - centraux aux projets féministes québécois qui semblent être produits (et maintenus) par le système politique libéral actuel et ses bases idéologiques.

Tout d'abord, Monique Lanoix propose de façon fort convaincante d'utiliser l'éthique du care pour définir les soins de base que l'État doit minimalement fournir aux personnes âgées. Lanoix réussit ainsi à dépasser deux difficultés rencontrées par la théorie du prudential lifespan account de Norman Daniels. Elle assure de la sorte que, d'une part, les personnes atteintes de démence puissent être considérées comme des sujets de la justice (et ainsi qu'on leur garantisse l'accès à des soins de santé) et que, d'autre part, soit reconnue la complexité relationnelle des soins auxiliaires essentiels fournis par les proches aidant.e.s afin de mieux apprécier le travail de ces dernier.ère.s, et de les soutenir adéquatement. Dans le contexte québécois actuel du vieillissement de la population, Lanoix propose ainsi un projet politique spécialement pertinent. 
Agnès Berthelot-Raffard, pour sa part, nous propose, comme le titre de son chapitre l'indique, de repenser "le care comme cœur de la justice". L'un des principaux objectifs de l'éthique du care est de revaloriser l'aide informelle et les soins fournis dans la sphère privée. C'est donc dans cette optique que Berthelot-Raffard cherche à clarifier le statut de l'institution «de la vie ordinaire» isolée dans la sphère privée qui comporte une structure hiérarchique à la base de plusieurs inégalités et rapports d'oppression rarement questionnés politiquement: la famille. Dans un premier temps, Berthelot-Raffard examine la théorie de la justice sociale de John Rawls qui, selon elle, en niant la complémentarité entre la famille (institution «naturelle» selon Rawls) et les autres institutions (publiques), sépare clairement les questions d'ordre moral et de celles d'ordre politique, et se retrouve conséquemment à distinguer les sujets de besoins et ceux de droits. L'auteure nous invite alors à intégrer le care et la dépendance à la notion de justice afin de placer l'aide et les soins fournis dans la famille au cœur des questions sociales (collectives). En nous proposant de reconnaître la nature politique de la famille afin de régir cette institution par une éthique de justice, Berthelot-Raffard présente implicitement une réflexion sur la place des femmes au sein de la famille et de la sphère privée. Elle met ainsi en lumière à la fois la charge politique de l'éthique du care et sa critique féministe.

Dans le chapitre suivant, Stéphanie Gaudet développe une conception de la participation sociale ancrée dans les éthiques du care, et sur le don. Afin de saisir dans sa complexité l'expérience réelle de la participation sociale, Gaudet nous propose de renouveler notre compréhension de la participation sociale, de la société et du citoyen en arrêtant de distinguer ce qui relève de la participation politique et civique (les pratiques formelles) des actions d'ordre privé (les pratiques informelles). Les responsabilités civiques s'inscrivant dans les relations qui relient les individus les uns aux autres en tant qu'aidants, la participation devrait s'analyser à partir de ce que Gaudet appelle «l'interstice entre don et care». Dans ce texte, Gaudet nous présente donc une réflexion sur les structures de participation sociale. Toutefois, la longue présentation sociologique sur le social, la participation, le don et le care semble quelque peu incomplète puisqu'elle n'est ni rattachée à une proposition politique établissant clairement la pertinence pratique de cette analyse renouvelée de la participation sociale, ni reliée à des moyens concrets d'opérer un changement dans l'analyse des pratiques sociales afin d'avoir une compréhension intégrée du public et du privé.

Sophie Bourgault conclut la deuxième partie de l'ouvrage avec un texte soulignant la place centrale de l'écoute et de l'attention dans l'éthique du care. Elle nous invite à dépasser la dichotomie parole/silence à laquelle on associe traditionnellement l'opposition liberté/obéissance. Même si l'émancipation des femmes du patriarcat passe, entre autres, par la prise de parole, Bourgault veut repenser cette vieille dichotomie aristotélicienne entre la parole et le silence qui renforce l'invisibilité des individus les plus marginaux qui n'ar- 
rivent pas à se faire entendre. En se référant aux écrits de Simone Weil, Bourgault demande de «baisser le volume » des discours dominants afin de pouvoir porter notre attention sur les questions souvent laissées dans l'ombre. Accepter les vulnérabilités et rendre visibles les inégalités demande qu'on écoute les "dépendants» et qu'on soit attentif à leurs besoins. On peut alors aborder de front des enjeux de justice sociale de manière inclusive et concertée. Cette proposition de Bourgault permet d'imaginer une dynamique politique (et plus largement un espace public) plus ouverte et respectueuse des différences dans laquelle pourrait se déployer à son plein potentiel une politique du care.

Finalement, dans la partie "Le jugement, l'intersubjectivité, la vulnérabilité en question", quatre auteures nous proposent des réponses à des enjeux philosophiques actuels à partir des réflexions du care. À travers ces dialogues entre l'éthique du care et les traditions occidentale et japonaise, la portée théorique et réflective de l'éthique du care est encore une fois élargie. Sur le plan philosophique, le care ne se présente plus comme une simple rupture avec la tradition, mais plutôt comme un outil réflexif permettant de revisiter des questions philosophiques contemporaines. La charge critique féministe du care apporte en effet un regard différent sur des questions pouvant dès lors être réactualisées (et non mises de côté).

Sophie Cloutier présente une lecture comparative du concept de jugement politique chez Joan Tronto et Hannah Arendt. Même si Arendt faisait des soins et de la sphère privée des questions prépolitiques, son concept d'amor mundi ("souci pour le monde") n'est toutefois pas sans rappeler les réflexions de Tronto sur les soins. En effet, Arendt refusait le modèle de l'autonomie complète du sujet, et ce surtout dans le domaine politique. Elle mettait plutôt l'accent sur la notion de pluralité chez Kant afin de développer les concepts d'intersubjectivité et de mentalité élargie: deux concepts également centraux à la définition du care de Tronto. Ce lien entre les théories d'Arendt et de Tronto ouvre la porte, dans les théories du jugement politique, à un déplacement du paradigme libéral de l'indépendance vers celui de la vulnérabilité et des relations. Cloutier positionne ainsi habilement l'éthique du care comme une nouvelle étape dans la réflexion politique, et non comme une proposition en marge de la tradition philosophique ou parallèle à celle-ci.

Dans son texte, Marjolaine Deschênes se penche sur les analyses littéraires de Gilligan dans Une voix différente qui font, selon elle, apparaître les variations autour de l'invariant du temps historique. À partir de là, Deschênes présente le concept de "littérature care» qui reprend les concepts de récit et d'identité narrative chez Paul Ricoeur. Elle souhaite ainsi articuler les bases d'un courant littéraire - encore peu étudié — qui met en danger le discours dominant en se concentrant sur les identités singulières, les vulnérables et les fragiles. L'originalité de ce chapitre montre bien l'étendue de l'intérêt de l'éthique du care. Il permet également de reconnaître que, malgré les nombreuses critiques adressées à l'œuvre de Gilligan, entre autres par des féministes, cette œuvre renferme encore un potentiel réflexif appelant à être développé. 
Bettina Bergo mobilise pour sa part la notion husserlienne d'empathie radicalisée par l'idée de "l'autre-dans-le-même» de Lévinas, de manière à développer et justifier la théorie relationnelle qu'est l'éthique du care. Bergo distingue en effet au cœur même de la phénoménologie du $\mathrm{Xx}^{\mathrm{e}}$ siècle les fondements théoriques du care. Elle nous présente donc le care comme une théorie qui renouvelle certaines réflexions philosophiques contemporaines en phénoménologie. Le chapitre de Bergo s'intègre ainsi fort pertinemment dans l'objectif réflectif de la troisième partie de l'ouvrage. Toutefois, sa complexité théorique rend la compréhension de la proposition de Bergo un peu moins accessible que celle des autres textes de l'ouvrage.

Finalement, Mariko Konishi conclut l'ouvrage par une réflexion comparative entre l'éthique du care et le concept japonais de l'amae. Konishi nous explique que si la proposition de l'éthique du care de reconnaître la condition de dépendance qui unit les individus est déstabilisante pour plusieurs occidentaux, elle semble toutefois aller déjà de soi pour les Japonais. En effet, au Japon, les relations intimes de "codépendance» entre deux adultes n'ont pas toujours une connotation péjorative, bien au contraire. Ce que l'éthique du care pourrait considérer comme une situation abusive ne l'est pas nécessairement du point de vue de l'amae. On comprend ainsi que le care renvoie à l'expérience occidentale des soins et des relations familiales. Le pas de recul que nous offre Konishi nous fait réaliser que, même en élaborant une théorie éthique particulariste, celle-ci demeure porteuse du contexte sociohistorique dans laquelle elle se développe: les critères de validité éthique d'une relation de "codépendance» s'établissent directement en fonction du contexte social dans laquelle celle-ci se déploie. Le texte de Konishi nous rappelle donc de ne pas attribuer un caractère universel à la façon dont les situations d'interdépendance sont vécues et jugées en Occident.

En somme, l'ouvrage La care. Théorie éthique féministe pose des bases théoriques solides à une éthique du care dé-genrée et dé-sentimentalisée afin de relancer à nouveaux frais de nombreuses réflexions politiques et philosophiques. Il s'agit tout autant d'un livre d'introduction (dans la mesure où la première partie permet de définir clairement l'éthique du care, son contexte d'élaboration ainsi que ses principaux intérêts critiques), que d'un ouvrage offrant un déploiement réflexif et critique important du concept de care à travers une variété de questionnements et d'enjeux contemporains.

Notons également que le projet commun partagé dans les articles que regroupe l'ouvrage dirigé par Bourgeault et Perreault donne un caractère complémentaire à l'ensemble des réflexions qui nous y sont présentées. Ainsi, malgré la diversité des sujets abordés, et parfois la portée inégale des propositions qui y sont faites, cet ouvrage est un ajout essentiel à la littérature foisonnante sur l'éthique du care. 